\section{Ärztegesellschaft des Kantons Bern}

Medizinischer Bezirksverein Bern-Stadt

Zur Aufnahme als ordentliche praktizierende Mitglieder haben sich angemeldet:

med. prakt. Dominic Naville, Frikartweg 21, 3006 Bern

Dr. med. Martin Zingg, Mattenhofstrasse 22, 3007 Bern

Einsprachen gegen dieses Vorhaben müssen innerhalb von 14 Tagen seit dieser Veröffentlichung schriftlich und begründet dem Präsidenten des Medizinischen Bezirksvereins Bern-Stadt eingereicht werden. Nach Ablauf der Einsprachefrist entscheidet der Vorstand über die Aufnahmen der Gesuche und allfällige Einsprachen.

Der Vorstand des MBV Bern-Stadt

\section{Ärztegesellschaft Thurgau}

Zum Eintritt in die Ärztegesellschaft Thurgau hat sich angemeldet:

Dr. med. Swen Galda, Facharzt für Allgemeine Medizin und für Psychiatrie und Psychotherapie, Assistenzarzt Liaisonpsychiatrie in der Klinik für Kinder und Jugendliche des Kantonsspitals Münsterlingen.

Einsprachen gegen die Aufnahme sind innerhalb von 10 Tagen seit der Publikation beim unterzeichneten Sekretariat schriftlich zu erheben.

Ärztegesellschaft Thurgau Der Sekretär: Robert Fürer

\title{
Bekanntmachungen / Communications
}

\section{Schweizerische Gesellschaft für Endokrinologie und Diabetologie \\ Société Suisse d'Endocrinologie et de Diabétologie}

Vorstand/Comité 2002

Präsident/Président:

Prof. Giatgen A. Spinas, Zürich

Vizepräsident/Vice-président:

PD Dr. Peter Diem, Bern

Sekretär/Secrétaire:

Dr. Marius Kränzlin, Basel

Kassier/Trésorier:

Dr. Rolf Gräni, Wolhusen

Beisitzer/Membres:

Dr François Kuntschen, Monthey (Präsident der Ärztekommission der SDG);

Dr. Madeleine Straumann, Olten;

Prof. Ulrich Keller, Basel;

Prof. Primus Mullis, Bern;

Prof. Eugen Schönle, Zürich;

PD Dr François Pralong, Lausanne;

PD Dr Michel Rossier, Genève;

PD Dr Christoph A. Meier, Genève;

Dr. Lukas Villiger, Baden;

Dr. Jan Komor, Bern.

\section{Schweizerische Gesellschaft für Viszeralchirurgie \\ Société Suisse de Chirurgie viscérale}

Vorstand/Comité 2002

Präsident/Président:

Prof. Dr Philippe Morel, Genève

Vizepräsident/Vice-président:

PD Dr Marco Merlini, La Chaux-de-Fonds

Schriftführer/Secrétaire:

Prof. Dr. med. Felix Largiadèr, Erlenbach

Kassenführer/Caissier:

Dr. med. Rainer Hoffmann, Aarau

Beisitzer/Assesseurs:

Dr. med. Jean-Pierre Barras, Solothurn;

Prof. Dr. med. Philippe Gertsch, Bellinzona;

Prof. Dr. med. Urs Metzger, Zürich;

Prof. Dr François Mosimann, Lausanne;

Dr Andreas Scheiwiller, Genève;

PD Dr. med. Hans E. Wagner, Thun;

Dr. med. Markus Weber, Zürich. 